\title{
Potentialités de l'écotourisme et géotourisme aux provinces de Tantan, Tarfaya et Layoune (Sahara Atlantique marocain)
}

\author{
Taha Amimi, Doctorant \\ Radouane Ouhnine, Doctorant \\ Jamal Chao, Professeur
}

Université Ibn Tofail/Faculté des Sciences/Département de Géologie/Laboratoire Géosciences des Ressources Naturelles/Kénitra, Maroc.

Hicham Elbelrhiti, PhD. Professeur

Institut Agronomique et Vétérinaire Hassan II/Département des

Sciences Fondamentales et Appliquées/Rabat, Maroc.

Avy Stéphane Koffi, PhD. Assistant

Université Péléforo Gon Coulibaly de Korhogo/Département de

Géoscience/Côte d'Ivoire

doi: 10.19044/esj.2017.v13n15p133 URL:http://dx.doi.org/10.19044/esj.2017.v13n15p133

Abstract

Tantan, Tarfaya and Laayoune provinces, which belong to the Moroccan Atlantic Sahara, are characterized by exceptional geo and ecotourism potentials. In this context, singing dunes, sand rivers and the presence of several wadis mouth's in such desert environment could constitute a locomotive for the development of green and geological tourism. Indeed, the studied region presents Eco and Geo-tourism potentials, combining ecological sites classified by the RAMSAR convention as the Khnifis lagoon, the wadis mouth's including the longest river in Morocco (Oued Draa) with its new national park. Rivers of sand with multitudes sand colours, singing dunes, virgin beaches and sebkhas with the lowest point in Morocco, the sebkha of Tah with an altitude of $-55 \mathrm{~m}$ adsl.

Keywords : Eco-tourism, Geo-tourism, Potentialities, Moroccan Atlantic Sahara

Résumé

Les provinces de Tantan, Tarfaya et de Laâyoune qui font partie du Sahara Atlantique marocain se caractérisent par des potentialités touristiques, 
géologiques et écologiques d'une qualité exceptionnelle. Dans ce cadre, les dunes chantantes, les fleuves de sable et la présence de plusieurs embouchures dans un tel milieu désertique pourraient constituer une locomotive pour le développement du tourisme vert et géologique dans cette région. En effet, la région présente des potentialités éco et géotouristiques mêlant des sites écologiques classés par la convention RAMSAR comme la lagune de Khnifis, des embouchures des oueds dont celle du plus long fleuve au Maroc (Oued Draa) avec son nouveau parc national. Les fleuves de sables constitués par les dunes chantantes, les multitudes couleurs de dunes, les plages et les sebkhas avec le point le plus bas au Maroc, situé au niveau de la sebkha de Tah (-55 m d'altitude) pourraient être considérés comme des potentialités éco-géotouristiques prometteuses.

Mots-clés : Ecotourisme, Géotourisme, Potentialités, Sahara Atlantique Marocain

\section{Introduction}

L'écotourisme ou le tourisme vert est défini comme une des formes $\mathrm{du}$ tourisme durable. Il est plus centré sur la découverte de la nature (écosystèmes, agrosystèmes, tourisme rural...etc.), voire d'écologie urbaine (réserves naturelles urbaines, espaces verts écologiques, jardins écologiques ...etc.). Il est défini par la Société International de l'Ecotourisme (TIES) comme un voyage responsable dans des environnements naturels où les ressources et le bien être des populations sont préservés. Quant au géotourisme, c'est un concept introduit par la National Geographic Society dont elle détient toujours l'appellation (National Geographic, 2010). Elle le définit comme un tourisme qui soutient et met en valeur une destination selon son caractère géographique, son environnement, sa culture, son esthétisme, son patrimoine et le bien-être de ses habitants. Donc c'est un tourisme qui préserve et valorise le caractère géographique d'un lieu et qui englobe les principes de développement durable.

L'écotourisme et le géotourisme sont en pleine expansion partout dans le monde et sont devenus de plus en plus d'actualité à l'échelle mondiale. Certains ont fait de ces types de tourisme un moteur pour le développement touristique. A titre d'exemple, la ville de Montréal au Canada a crée une charte appelée Géo-tourisme Montréal. Cette charte de géotourisme a été signée par trois organisations qui composent son exécutif : Héritage Montréal, Tourisme Montréal et le Centre mondial d'Excellence des Destinations (CED) (Charte de géotourisme, 2007). Au Maroc, on note quelques expériences réussies à Marrakech, Mirleft, Ifrane, Ouarzazate et Merzouga qui ont plus au moins réussi à intégrer les potentialités éco et géotouristiques de l'Atlas et du Sahara dans leurs circuits. A titre d'exemple, 
les paysages de l'Anti-Atlas sont visités par 3 à 5 millions de touristes par an (El Hadi et al., 2011). Toutefois la partie du Sahara Atlantique marocain reste en dehors du boom du tourisme vert et responsable malgré la présence des sites d'une richesse exceptionnelle et une culture séculaire.

Ces dernières années, l'état marocain a consacré des moyens lourds pour développer le tourisme dans ces régions. Dans ce cadre, il a réactivé le moussem des nomades de Tantan, le port de Tarfaya est actuellement ouvert aux ferries avec les Iles des Canaries, il a assuré l'ouverture de l'aéroport de Tantan et Guelmim aux vols nationaux vers Agadir et Casablanca. Également, le gouvernement a lancé un projet pour créer une station touristique balnéaire à Oued Chbika au Sud de Tantan. Ces efforts ne tiennent pas compte les potentialités du tourisme culturel, vert et géologique dans les plans de développement du tourisme dans la région. Dans ce cadre, la richesse et la diversité des traditions des habitants de la région et la variabilité des sites naturels méritent amplement d'être valorisées et intégrées dans de nouveaux circuits très prometteurs et qui concordent avec la stratégie du pays en matière de tourisme dans sa vision 2020. En effet, en plus des traditions ancestrales, ces provinces se caractérisent par des potentialités touristiques, géologiques et écologiques d'une qualité exceptionnelle. Ainsi, les dunes chantantes, les fleuves de sable et la présence de plusieurs embouchures dans un tel milieu désertique pourraient constituer des sites potentiels pour le développement du tourisme géologique vert et responsable.

La valorisation de ce potentiel pourrait être faite par l'inventaire, la cartographie, le développement des circuits balisés et le classement de quelques sites comme étant des géomorphosites et géosites. Cette valorisation pourrait contribuer à la création de l'appellation géotourisme Sahara Atlantique. Dans cet article, nous présentons l'inventaire des potentialités de la région pour le développement de l'éco-géotourisme au Sahara Atlantique marocain.

\section{Situation, milieu physique et cadre administratif}

Les provinces de Tantan, Tarfaya et Laâyoune (Sud-Ouest marocain) sont situées au nord-est du Sahara et présentent différents aspects géomorphologiques (Figure 1). Cette partie vise donc à présenter les différentes composantes géologiques et géomorphologiques de la région étudiée. Toutefois, le lecteur pourra, s'il le souhaite, se référer à de nombreuses études et publications réalisées par des scientifiques dans lesquelles sont détaillées les différentes aspects géologiques de la région (Ortleib, 1975 ; Oulehri, 1992 ; Amri, 1998 ; Kabbachi, 2001 ; Elbelrhiti, 2005). 


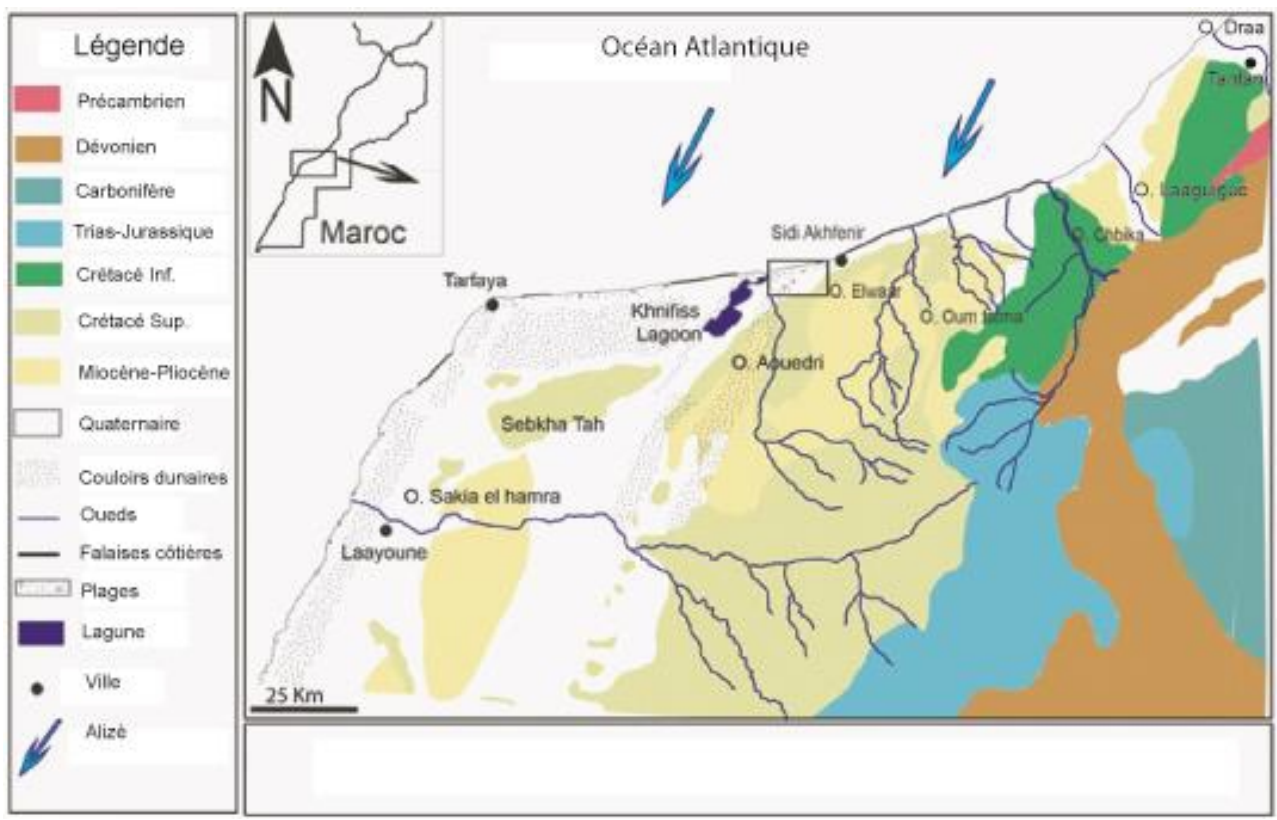

Figure 1: Carte simplifiée de la région étudiée montrant sa situation géographique et ses différentes formations géologiques

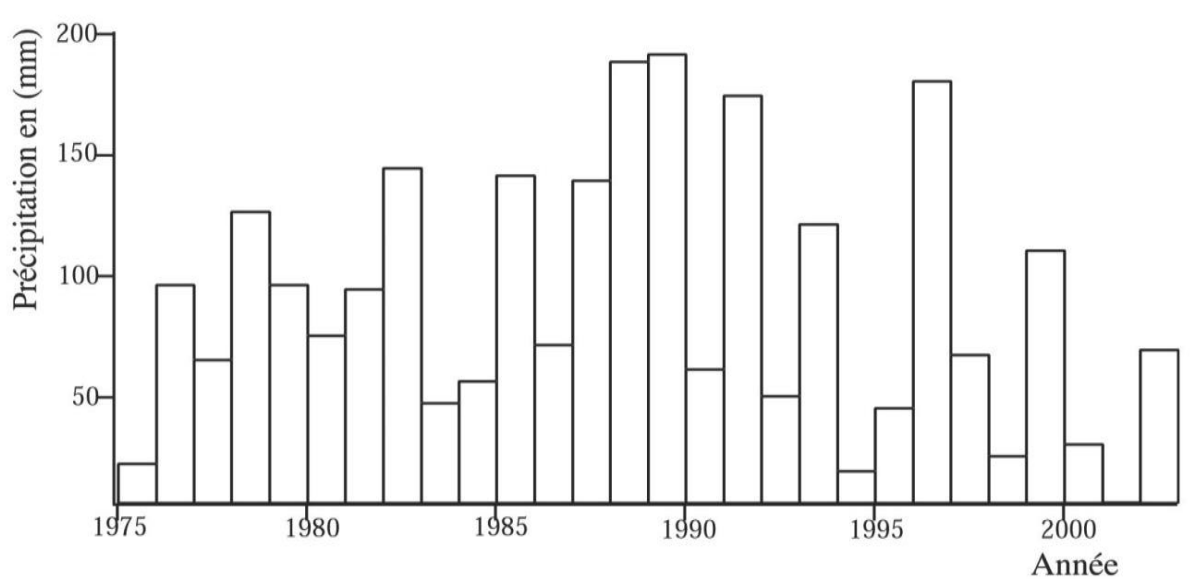

Figure 2 : Précipitations enregistrées à Tantan entre 1975 et 2003

\section{Climat et couvert végétal}

Le climat de la région d'étude s'inscrit dans le type saharien à hiver chaud selon la classification d'Emberger (Emberger, 1955). Il est caractérisé par de faibles précipitations (Figure 2) et des températures moyennes douces (Delannoy, 1975 ; Żmudzka et al., 2014). Cependant, cette classification néglige la position géographique de la région qui fait partie du domaine boréal de l'alizé maritime généralement humide et entraînant de faibles écarts thermiques (Leroux, 1983). Ces alizés sont parmi les vents les plus 
uni-modale au monde avec un indice RDP/DP = 0,91 (Elbelrhiti et al., 2005). Autrement dit, il y a une direction dominante des vents de la région. Cette situation particulière du Sahara Atlantique donne à la région un climat très original. Cette particularité se traduit biologiquement par la présence de plantes endémiques, une flore très spéciale comprenant des éléments tropicaux et en particulier des euphorbes cactoïdes et arborescentes délimitant au Maroc une zone dénommée par les phytogéographes « enclave macaronésienne marocaine » (Emberger, 1955 ; Médail \& Quézel, 1999). D'un autre coté, sur les dolines argileuses, se développe une végétation arbustive et ligneuse, un peu plus variées. Aussi, sur les zones humides telles que les embouchures des oueds et les lagunes, se développent une végétation constituée essentiellement des salicornes.

\section{Géologie et géomorphologie}

Au sud de Tantan, s'étale une surface tabulaire quasi-plate ayant une pente générale de 1'ordre de $1 \%$ qui augmente graduellement en allant de l'Ouest vers l'Est. Ce plateau occupant une grande superficie est constitué par une dalle calcaro-gréseuse dure correspondant à l'étage du Moghrébien (Figure 1). Cette dalle constitue une couche protectrice des formations marno-calcaires tendre du Crétacé (Choubert et al., 1966). La disposition des couches dures sur couches tendres, la prédominance du calcaire et le régime du vent prépondérant sont à l'origine de l'abondance de plusieurs chottes et sebkhas (Kabbachi, 2001), dont la sebkha de Tah qui est considérée comme le point le plus bas au Maroc avec une altitude de $-55 \mathrm{~m}$ sous le niveau de la mer (Choubert et al., 1966).

L'interaction de ce plateau avec l'océan se fait au Nord par des falaises abruptes et à partir d'AKhfenir par des plages intercalées par quelques falaises (Figure 3 a et b) (Oulehri, 1992). Des flèches littorales prennent naissance tout au long des falaises et s'organisent en véritables cordons dunaires à l'embouchure des oueds (Figure $3 \mathrm{a}$, b et d) (Abia et al., 2009). Sur les plages, l'abondance du sable amené par ces flèches littorales donne naissance à des immenses champs de dunes qui s'étalent sur des surfaces qui peuvent aller jusqu'à $300 \mathrm{~km}$ de long et seulement une dizaine de kilomètre de large (Elbelrhiti, 2005). Ceci explique l'appellation poétique de fleuve de sable donné par Arceduc (1967) à ces champs des dunes.

$\mathrm{Au}$ nord de Tantan, s'élève une zone montagneuse qui est la terminaison de la chaîne Anti-atlasique. Sur la rive gauche de Draa apparaît son noyau précambrien (massif du bas Draa). Vers le Sud, cette chaîne est formée de formations cambriennes et ordoviciennes (Jbel Zini) plissées par les mouvements hercyniens qui s'ennoient sous les terrains crétacés. 


\section{Cadre administratif et socioéconomique}

De point de vue administratif, la province de Tantan fait partie de la région de Guelmim- Oued Noun alors que les deux autres provinces; Tarfaya et Laâyoune font partie de la région de Laâyoune-Sakia El Hamra. Selon le recensement établi par le Haut Commissariat au Plan du Maroc en 2014, 238096 habitants ont été recensés à la ville de Laâyoune, 13082 à la ville de Tarfaya et 86134 à Tantan. La densité de population de la région étudiée est parmi les plus faibles au Maroc. A titre d'exemple la province de Tantan qui compte une population de 86134 habitants pour une superficie $17295 \mathrm{Km}^{2}$, ce qui une densité de 4,98 habitants $/ \mathrm{km}^{2}$. La pêche, l'élevage et l'artisanat en plus des mines sont les principales ressources des trois provinces. Le tourisme et l'agriculture viennent en deuxième position (El Mahdad et Ouhajou, 2007).

Pour le secteur du tourisme, malgré les potentialités de la région, les incidences de l'activité touristique, sur l'économie de la région, restent encore limitées. À titre d'exemple, la structure d'accueil de la province de Tantan ne dépassait pas 39 hôtels en 2001 dont seulement 3 qui sont classés, plus un camping. Le nombre total de lits atteignait 876, dont seulement 101 classés. Les nuitées enregistrées en 2000 étaient au nombre de 15000 environ, dont $2 \%$ seulement de touristes étrangers et la durée moyenne de séjour ne dépassait pas une nuitée (El Mahdad et Ouhajou, 2007). Quant à la province de Laâyoune, la structure d'accueil ne dépassait pas 53 hôtels en 2013 dont seulement 26 qui sont classés. Le nombre total des lits atteignait 2538, dont 1587 classés. Les nuitées enregistrées en 2013 étaient au nombre de 33733 , dont $45.25 \%$ de touristes étrangers et la durée moyenne de séjour ne dépassait pas deux nuitées (Annuaire Statistique Régional de LaâyouneBoujdour-Sakia El Hamra, 2014).

Signalons aussi que ces dernières années, ces provinces ont connu une croissance de flux de touristes qui prennent l'aventure de suivre l'itinéraire du raid Paris-Dakar et du tourisme caravanier et dont l'incidence sur l'économie locale reste très limitée.

\section{Identification des potentialités éco et géotouristiques}

Les trois provinces qui constituent notre région d'étude offrent des potentialités exceptionnelles pour développer l'écotourisme et le géotourisme.

\section{Le chant et les champs des dunes}

L'émission spontanée de son par les dunes connue communément par le chant de dunes a fasciné de nombreux explorateurs dont Marco Polo (1298). Il comparait ce son à un concert d'instruments de musique et le liait à des saints-esprits. Cette énigme du chant des dunes est due probablement à la 
rareté des dunes chantantes. En effet, seule une quarantaine de dunes au monde seraient capables de chanter dont les trois mégabarkhanes de la région étudiée (Andreotti, 2004 ; Douady et al., 2006). Ce chant de dunes pourrait constituer un attrait particulier pour les touristes.

Comme nous avons évoqué précédemment, les champs des dunes de la région se présentent sous forme de fleuves de sable. En effet, dans la région il y en a trois (Figure 4):

* Le premier fleuve de sable prend naissance à Foum Agoutir (ancien Peurto Consado) jusqu'au Sakia El Hamra. Il a une direction NNE-SSW et une longueur de $150 \mathrm{Km}$.

*Le second est le plus court, il fait un peu plus de $40 \mathrm{Km}$ de long. Ce fleuve nait à quelques dizaines de kilomètres de Tarfaya et meurt à la sebkha de Tah.

* Le troisième est le plus intéressant et le plus long. Il a une longueur de 310 $\mathrm{km}$, commence à Cap Juby (4 Km au N-E de Tarfaya) et dépasse Laâyoune par $200 \mathrm{~km}$.

De point de vue géomorphologique, tous les travaux qui ont été faits dans la région s'accordent sur le fait que la dune barkhane est la forme dunaire dominante dans la région (Pacheco, 1946 ; Arceduc, 1967 ; Oulehri, 1992 ; Kabbachi, 2001 ; Sauermann et al 2000; Hersen et al 2004 ; Elbelrhiti, 2005).

Mais ce type de formation sableuse n'est pas le seul présent. En effet, différentes formes d'accumulations de sables cohabitent et partagent le même endroit avec la dune en croissant (Figure $3 \mathrm{c}$, e et f): 

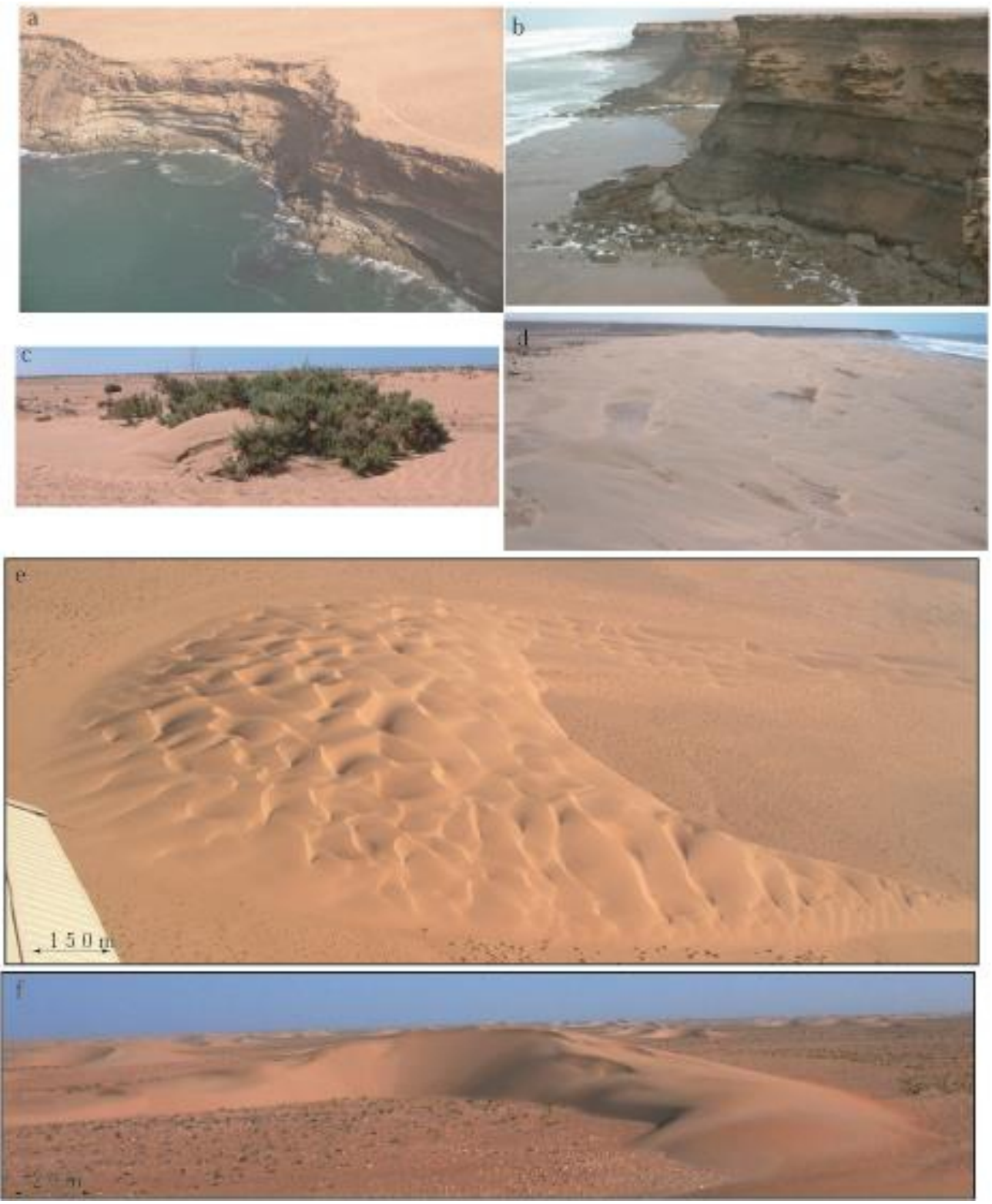

Figure 3 : a et b) Des falaises au bord de l'Océan Atlantique. c) Photo d'une nebkha au sein du premier fleuve de sable. d) Embouchure de l'Oued Chbika avec des dunes transverses. e) Mégabarkhane aux alentours de la lagune de Khnifiss. d) Barkhane typique de la région. 


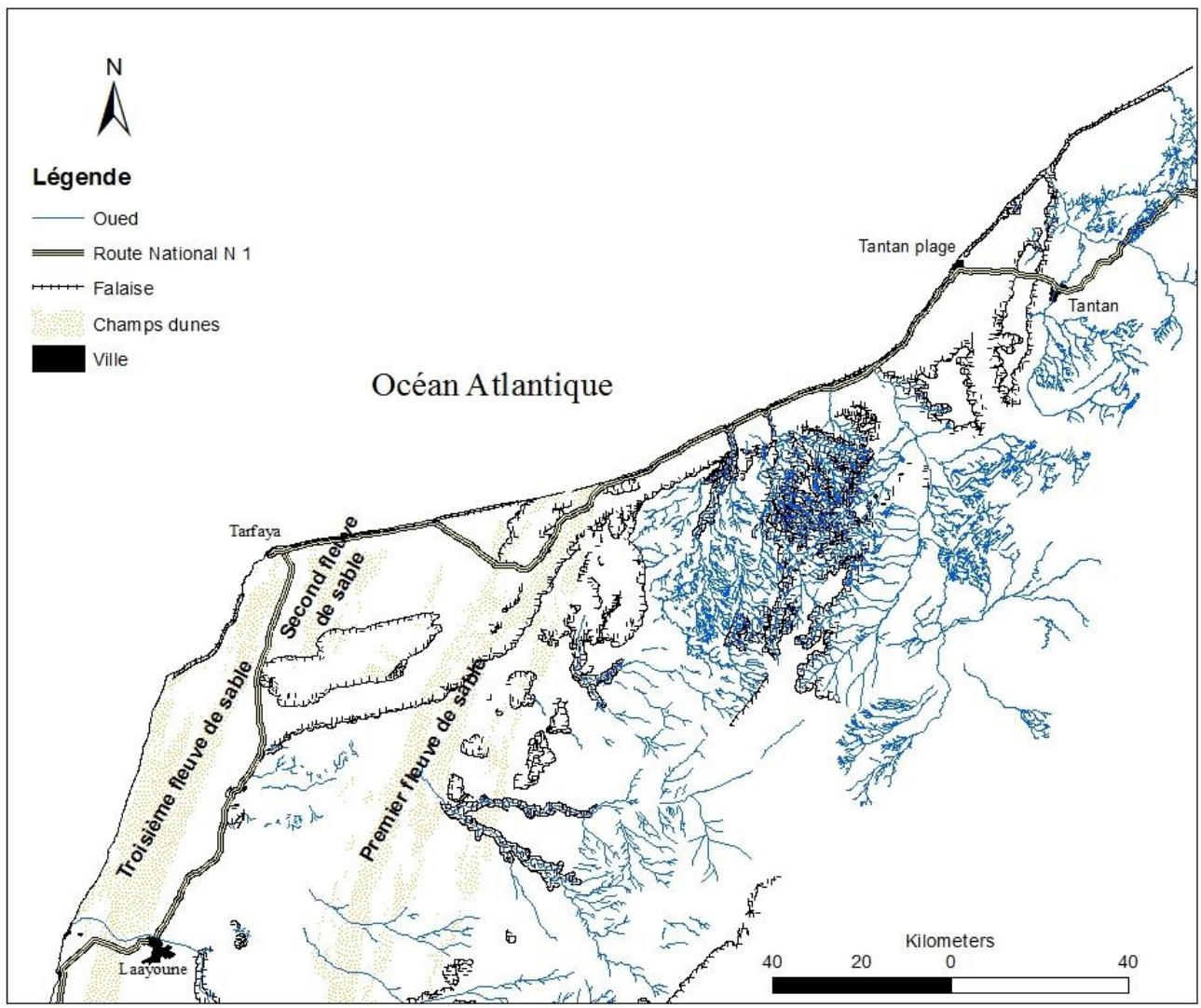

Figure 4 : Carte montrant les trois fleuves de sable.

- $\quad$ Les barkhanes et les dunes transverses :

La barkhane est le type de dune le plus simple et le plus étudié. Elle a une forme en croissant et nait quand le vent efficace prend constamment la même direction tout au long de l'année et s'il n'y a pas assez de sable pour recouvrir la totalité de la surface de la terre (Elbelrhiti \& Hargitai, 2014). Les dunes transverses naissent dans les mêmes conditions de vent que les barkhanes mais avec une alimentation en sable beaucoup plus forte. En d'autres termes, les dunes transverses sont le résultat de la coalescence des barkhanes suite à l'abondance du sable (Fryberger \& Dyne, 1979).

- $\quad$ Les Mégabarkhanes ou Mégadunes

Les mégabarkhanes est comme indique leur nom, sont des dunes géantes en forme de croissant. Pour fixer les idées, l'une des mégabarkhanes de la région fait 40 mètres de haut et 650 mètres de large alors qu'une grosse barkhane fait 10 à 12 mètres de haut et 120 à 150 mètres de large. En plus, ces dunes présentent la particularité d'avoir de petites barkhanes dans leurs sillages. Dans le bassin Tarfaya-Laâyoune se trouve trois mégabarkhanes à une dizaine de kilomètres au Sud-Ouest de Sidi Akhfénir. Ces mégadunes présentent la particularité d'avoir la couleur rouge par rapport aux autres 
dunes qui ont plutôt une couleur jaune blanchâtre (Moustakim, 2014 ; Adnani et al., 2015).

- La nebka

Le mot nebka est d'origine arabe désignant les sédiments (généralement sablonneux) qui s'accumulent derrière des obstacles comme les végétaux (Necsoiu \& Hooper, 2014). Dans les fleuves du sable et à côté des mégadunes, on trouve de petites nebkas de l'ordre d'une dizaine de centimètres de haut. C'est ce type de nebka qui est prépondérant dans la région. Quant aux grosses nebkas telles quelles sont décrites dans la littérature, elles poussent derrières des plantes qui peuvent dépasser le 1 mètre de haut comme le zizyphus lotus. Généralement, on les trouve à la plage de Mégrio et dans les endroits humides à savoir les sebkhas et les Oueds (Elbelrhiti, 2005).

\section{La réserve biologique et le parc national}

La région qui nous intéresse est riche par son patrimoine écologique et biologique, d'où l'instauration d'un parc national : Le parc National de Khnifiss. Il est situé à une dizaine de kilomètres au Sud d'Akhfenir et dont la lagune est classée zone RAMSAR par l'UNESCO depuis 1980 et de ce fait elle est très connue à l'échelle national et international. Le parc de Khnifiss se caractérise par la variété de son environnement à la fois marin, lagunaire et désertique. La lagune s'étend sur $20 \mathrm{~km}$ de longueur et sur 6500 hectares parmi les 180000 hectares du parc national de Khnifiss. Dans cette réserve, les touristes peuvent regarder 179 espèces d'oiseaux (Fiche technique du SIBE 'Baie de Khnifiss', 2006). Il comprend six espèces de plantes d'importance mondiale. La lagune abrite également 30 espèces de macroalgues et 72 espèces de plantes vasculaires d'une richesse énorme en invertébrés marins dont 50 mollusques, 74 crustacés, 18 polychètes et 27 mammifères dont 2 sont endémiques (Fiche technique du SIBE ' Baie de Khnifiss', 2006).

Les valeurs naturelles du site sont valorisées par la présence de vestiges archéologiques préhistoriques constitués de silex taillés, fragments d'œufs d'autruches graves et perles de collier, en œufs d'autruches, tortues et monuments historiques comme les ruines d'une Tour portugaise (Charon et al., 1973).

Un projet pour la création d'un deuxième parc national dans la région est en cours. Il s'agit du parc du bas de Draa à l'Est de Tantan (Ledauphin, 2005). Ce parc qui est toujours à l'état de projet est constitué de vastes étendues d'Acacia raddiana associées à des Balanites et Tamarix et d'autres arbres sahariens très rares. Il abrite des espèces des plus rares et spectaculaires comme le Guépard, le Lynx caracal, la Gazelle de Cuvier, la gazelle dorcas, le Ratel, ainsi qu'une herpétofaune très riche représentée par 
le Caméléon, le Varan de désert, le Fouette-queue, le Naja et la Vipère de l'erg (Ledauphin, 2005).

\section{Les sebkhas et les hamadas}

Les sebkhas sont des dépressions sans écoulement, à fond plat et entourées de falaises à bord franches. En général leur fond est composé d'un dépôt salin et alimenté par des sources éphémères (Thomas \& Goudie, 2000). L'une des particularités de la région est l'existence de plusieurs sebkhas dont la sebkha de Tah qui est la plus importante et fait $30 \mathrm{Km}$ de long et $11 \mathrm{Km}$ de large. Elle est également le point le plus bas au Royaume (55m d'altitude) (Bouab, 2003).

\section{La pêche et les plages}

La pêche pourrait constituer un facteur potentiel pour attirer les touristes vers cette région vue l'étendue des côtes dont elle dispose. D'ailleurs et à cause des upwellings qui font remonter les eaux froides vers la surface et par conséquent les nutritifs et les planctons, ces cotes sont parmi les plus poissonneuses au monde (Gebhardt \& Zorn, 2008). Nous avons constaté que ces dernières années ce type de tourisme commence à attirer des investisseurs étrangers. Ainsi nous avons assisté à l'installation de deux établissements à Akhfenir intéressés par ce genre de tourisme. Toujours en relation avec l'océan, la région profite de plages très étendues que l'on peut subdiviser géographiquement en quatre parties (Oulehri, 1992) :

- Des plages des embouchures des oueds comme celle de Draa, Chbika et Elwaar (Figure $3 \mathrm{~d}$ ).

- Une plage qui prend naissance à Sidi Akhfenir et s'étale de part et d'autres de foum Agoutir.

- Une plage naissant à $20 \mathrm{~km}$ à l'Est de Tarfaya jusqu'au Cab Juby, elle est interrompue par un petit tronçon de falaise (Figure $3 \mathrm{a}$ et $\mathrm{b}$ ).

- Une plage commençant à Mégrio et allant jusqu'au foum Elouade et présentant la plage la plus étendue de la région.

Ces plages présentent des fluctuations de largeurs, ainsi elles sont de l'ordre de $20 \mathrm{~m}$ à Sidi Akhfénir qui représente la plage la plus restreinte et dépasse les $750 \mathrm{~m}$ à l'Oued Elwaâr qui représente la plage la plus large de la région (Oulehri, 1992).

\section{Couleurs des dunes de sable}

Les grains de sable et les dunes de la région présentent une multitude de formes et de couleurs. Les grains des mégabarkhanes ont la particularité d'avoir une couleur rouge par rapport aux autres dunes de la région qui ont une couleur jaune blanchâtre. Cette différence de couleur est attribuée au taux de calcaire faible sur le sable des mégadunes et relativement fort pour 
les dunes transverses. Ceci pourrait être à l'origine de l'aspect blanchâtre des dunes transverses. La couleur rouge des mégadunes est attribuée à l'oxydation du fer en oxyde de fer qui constitue une pellicule mince autour des grains de quartz (Elbelrhiti, 2005; Adnani et al., 2015). Le degré d'oxydation de fer dépend de la vitesse et la taille des dunes qui contrôlent le temps de résidence des grains de sable dans une dune (Elbelrhiti, 2005; Adnani et al., 2015). Ce temps de résidence est défini comme étant le temps nécessaire pour une dune à fin qu'elle traverse une distance équivalente à sa longueur. En effet, une mégabarkhane de la région fait $650 \mathrm{~m}$ de long et une vitesse de $2 \mathrm{~m} / \mathrm{an}$ (Elbelrhiti et al. 2005), ce qui donne un temps de résidence de l'ordre de 325 ans. Par contre, une barkhane moyenne de la région fait $150 \mathrm{~m}$ de long et une vitesse de $7 \mathrm{~m} / \mathrm{an}$, ce qui donne en moyenne un temps de résidence de 21 ans. On peut conclure, qu'un grain de sable piégé par la mégabarkhane reste en moyenne 15 fois plus de temps qu'un autre piégé par une barkhane moyenne. En plus des couleurs de dunes mentionnées cidessus, il y a des grains de couleur noir à base de magnétite qui apparaît sur les côtes à quelques kilomètres au Sud de Sidi Akhfénir (Elbelrhiti, 2005 ; Abia et al., 2009).

\section{Conclusion et perspectives}

Il ressort de notre description de la région étudiée que toutes les conditions naturelles sont favorables pour le développement d'un tourisme vert, écologique et géologique. Dans ce cadre, l'offre touristique des provinces de Tantan, Tarfaya et Laâyoune pourrait constituer une locomotive de développement si elle intégrait les différentes potentialités dont elles disposent. Ainsi, les étendues désertiques, les réserves biologiques, les sebkhas, les dunes et les plages devraient être préservées, valorisées et protégées. Cependant, une charte dénommée Géo-tourisme Sahara Atlantique marocain apparait nécessaire à mettre en place. Tous ces atouts, peuvent être une plateforme de développement d'un tourisme vert, responsable, durable et équitable.

\section{References :}

1. Abia, E.H. Ibhi, A. Nachit, H. Kabbachi, B. Nouidar, M. Ezaidi, A. 2009. Les sables titanifères des plages atlantiques du S-O Marocain entre Laâouina et Tarfaya : caractérisation et origine. Notes et Mém. Serv. Géol. Maroc n530, pp. 9-18.

2. Adnani, M. Azzaoui, M. A. Elbelrhiti, H. Ahmamou, M. Masmoudi, Lh. 2015. Dunes around Khnifiss lagoon (Tarfaya, SW of Morocco): Composition, itinerary in dune fields, effects on dunes' colours and morphodynamic. "American Geophysical Union "AGU" Fall meeting » San Fransisco, California, USA. 
3. Amri. E. 1998. La lagune de Khnifiss (Littoral Atlantique du Sahara Marocain) : Analyse d'un écosystème original. These, Univ. Paris VI. 211 pages.

4. Andreotti, B. 2004. The Song of Dunes as a Wave-Particle Mode Locking. Phys. Rev. Lett. 93, 238001.

5. Annuaire Statistique Régional de Laâyoune-Boujdour-Sakia El Hamra, 2014, Haut commissariat au Plan, Royaume du Maroc, Direction Régionale de Laâyoune. 158 pages.

6. Arceduc. C. A. 1967. Les fleuves de sables entre le Maroc et la Mauritanie, et la théorie des barkhanes. C. R. Acad. Sci. Paris, série D, 261, pp. 3637- 3639.

7. Bouab, N. 2003. Application des méthodes de datation par luminescence optique a l'évolution des environnements désertiques. Sahara Occidental (Maroc) et Canaries Oriental (Espagne). These, Univ. Québec, Montréal, 232 pages.

8. Charon, M. Ortlieb, L. Petit-Maire, N. 1973. Occupation humaine holocène de la région du Cap Juby (Sud-Ouest Marocain). Bulletins et Mémoires de la Société d'anthropologie de Paris. Vol. $10 \mathrm{~N}$. 4, pp. 379-412.

9. Charte du Géotourisme. 2007, http://montrealmetropoleculturelle.org/pls/portal/docs/PAGE/BURE AU_MTROYAL_FR/MEDIA/DOCUMENTS/CHARTE\%20DU\%2 0G\%C9OTOURISME.PDF accès le 26/03/2017.

10. Choubert, G. Faure-Muret, A. \& Hottinger, L. 1966. Aperçu géologique du bassin côtier de Tarfaya. Notes et Mem. Serv. Geol., Maroc, 175/I.

11. Delannoy, H. Contribution à l'étude climatologique de la province de Tarfaya (Maroc). In : Contribution à l'étude scientifique de la province de Tarfaya. Trav. Inst. Chér., série générale, vol. 3 (1975) p. 15-60.

12. Douady, S. Manning, A. Hersen, P. Elbelrhiti, H. Protière, S. Daerr, A. and Kabbachi, B. 2006. Song of the dunes as a self-synchronized Instrument Phys. Rev. Lett. 97, 018002.

13. Elbelrhiti. H., 2005. Morphodynamique des barkhanes: Etude des dunes du Sud-Ouest Marocain. Thèse, Univ. Paris VII. 160 pages.

14. Elbelrhiti, H. Hargitai, H. 2014. Barchan in "Encyclopedia of Planetary Landforms » DOI 10.1007/978-1-4614-9213-9_12-1\# Springer Science+Business Media New York.

15. Elbelrhiti. H. Phillipe. P. and Andreotti B. 2005. Field evidence for surface-wave-induced instability of sand dunes. Nature 437 : pp. 720723. 
16. El Hadi, H. Tahiri, A. Simancas, J. F. González-Lodeiro, F. Azor, A. Martínez-Poyatos, D. 2011. Geoheritage in Morocco: The Neoproterozoic Ophiolite of Bou Azzer (Central Anti-Atlas). Geoheritage, 3:89-96. DOI 10.1007/s12371-011-0032-2.

17. El Mahdad, El H. et Ouhajou, L. 2007. Profil environnemental de Tantan. Projet Rapid Urban Sector Profiling for Sustainability UNHABITAT (ROAAS). 42 pages

18. Emberger, L. 1955. Une classification biogéoaraphique des climats. Rec. Trav. Lab. Bot. Géol. Fac. Se. 7(11): pp.3-43.

19. Fiche technique du SIBE ''Baie de Khnifiss', 2006. http://ma.chmcbd.net/manag cons/esp_prot/sibe ma/sibe lit/baie-dekhnifiss/sibe 136 accès le 19/01/2016.

20. Fryberger, S. G., and Deane, G. (1979) Dunes forms and wind regims. In McKee, E.D., ed, A study of global sand seas:US. Geol.Surv. Prof Paper 1052, p. 137-170.

21. Gebhardt, H. Zorn, I. 2008. Cenomanian ostracods of the Tarfaya upwelling region (Morocco) as palaeoenvironmental indicators. Revue de micropaleontologie, Vol. 51, issue 4, pp. 273-286. ISSN: 0035-1598 DOI: 10.1016/j.revmic.2007.02.001.

22. Hersen, P. Elbelrhiti, H. Andersen, K. H. Andreotti, B. Claudin, P. and Douady, S. (2004), Corridors of barchan dunes : stability and size selection, Phys. Rev. E, 69, 011304.

23. Kabbachi, B. El Youssi, M. Ezaidi, A. Rognon, P. 2001. Physiographie et dynamique sédimentaire actuelle dans la marge atlantique sud-ouest marocaine (Le bassin Tantan -Cap Juby). Quaternaire, Vol.12, No3. pp. 139-148.

24. Ledauphin, R. 2005. Les Vertébrés du Bas Draa (Sud Maroc) et leurs habitats. Rapport du Master, Univ. Paris XI. 47Pages.

25. Leroux, M. 1983 : Le climat de l'Afrique tropicale. Champion, Paris, 2 vols., 633 pp. and 24 pp. plus 250 maps.

26. Médail F. \& Quézel P., 1999 - The phytogeographical significance of South Western Morocco compared to the Canary Islands. Plant Ecology, vol 140, pp 221-244.

27. Moustakim, H. 2014. Apport de la télédétection spatiale et du SIG à la cartographie des Sols et l'étude de la morphodynamique des dunes de la lagune de Khnifiss (Sud-Ouest Marocain). Projet de Fin d'étude. Institut Agronomique et Vétérinaire Hassan II, 65 pages.

28. National Geographic. (2010). About geotourism. Retrieved September, 2015, from http://travel.nationalgeographic.com/travel/sustainable/about_geotour ism.html 
29. Necsoiu, M. \& Hooper,D. M. 2014. Nebkha in «Encyclopedia of Planetary Landforms » DOI 10.1007/978-1-4614-9213-9_12-1\# Springer Science+Business Media New York.

30. Ortleib, L. 1975. Recherches sur les formations plio-quaternaires du littoral Ouest-saharien: $\left(28^{\circ} 30^{\prime}-20^{\circ} 40^{\prime}\right.$ lat. N). Travaux et Documents de l'ORSTOM ; 48. ISBN 2-7099-0395-4. 268 pages.

31. Oulehri, T. 1992. Etude géodynamique des migrations de sable éoliens dans la région de Laâyoune (Nord du Sahara marocain), Thèse, Université paris VI.

32. Pacheco, H.P. 1946 Fases de formacion de los medanos en las playas de Cabo Juby. Bol. Soc. Esp. Hist. Nat. , XLIV. t1. pp. 239-242.

33. Polo, Marco, (1298), Le devisement du Monde, chap. LVII: De la cite de Lop, \& du grand desert (manuscript / printed ed. 1556, E. Groulleau, Paris, 144 p.: p. 24ab / 1938, The description of the world, translated and annotated by A.C. Moule \& Paul Pelliot, London: G. Routledge, 2 vol.).

34. Sauermann, G., P. Rognon, A. Poliakov, and H. J. Herrmann (2000), The shape of the barchan dunes of Southern Morocco, Geomorphology, vol. 36, pp.47-62.

35. Thomas, David G. and Goudie Andrew (2000), The Dictionary of Physical Geography, Blackwell publishing, 3rd ed.

36. Żmudzka, E. Woronko, D. Dłużewski, M. 2014. The Sources of moisture in the sand dunes - the example of the Western Sahara dune field. Quaestiones geographicae 33(3). pp 199-204. 